Paidéia, 2005, 15(32), 367-376

\title{
O TESTE DE ZULLIGER E AVALIAÇÃO DE DESEMPENHO ${ }^{1}$
}

\author{
Marcia Eloisa Avona Ferreira ${ }^{2}$ \\ Anna Elisa de Villemor-Amaral \\ Universidade São Francisco
}

\begin{abstract}
Resumo: Este trabalho aborda a avaliação da personalidade no contexto organizacional, tendo como objetivo buscar evidências de validade para o teste Zulliger por meio da correlação com um questionário de Avaliação de Desempenho. Foram avaliados os resultados de uma amostra de 86 sujeitos, com formação em Engenharia Elétrica, Engenharia da Computação, Engenharia Eletrônica, Ciência da Computação, Análise de Sistemas e Estatística, com idades entre 22 e 43 anos, gêneros masculino e feminino. O Zulliger foi analisado conforme o Sistema Compreensivo. A Avaliação de desempenho considerou os itens relacionamento interpessoal, tomada de decisões, busca por inovação, organização e solução de problemas. As análises de correlação entre os dois instrumentos indicaram que profissionais mais disciplinados e organizados, que possuem iniciativa e uma visão prática e buscam resultados concretos são mais bem avaliados do que os imaturos emocionalmente, altamente críticos e mais egocentrados, que priorizam as próprias necessidades em detrimento das dos outros.
\end{abstract}

Palavras-chave: Avaliação Psicológica; Zulliger; Sistema Compreensivo; Avaliação de Desempenho.

\section{THE ZULLIGER TEST AND LOB PERFORMANCE EVALUATION}

Abstract:This paper brings information about personality assessment in the organizational context, and the objective was to look for validity evidences for the Zulliger test in correlation with a questionnaire of Job Performance Evaluation. The results from a 86 subjects sample were examined. They had bachelor's degree in Electric Engineering, Computer Engineering, Electronic Engineering, Computer Science, System Analysis, and Statistics, men and women between 22 and 43 years old. The Z-test was analyzed using the Comprehensive System. The results showed that more disciplined and organized professionals, having initiative and practical vision, which target realistic results had an overall better evaluation. Not so well did the emotionally immature ones, or the ones highly critical and self-centered, who prioritize their own needs instead of the other ones' necessities.

Key words: Psychological assessment; Zulliger; Comprehensive System; Job Performance Evaluation.

Introdução: A avaliação psicológica é uma prática fundamental para profissionais de Psicologia em diversos ramos de atividade, pois possibilita um conhecimento maior da pessoa, o que embasará intervenções procedentes e adequadas. De acordo com Pasquali (2001), a avaliação visa, por meio dos mais variados métodos e técnicas, descrever e classificar o comportamento de uma pessoa, de modo a compreendê-la e tomar decisões, partindo-se do pressuposto de que a personalidade é aquilo que permite

\footnotetext{
${ }_{1}$ Artigo recebido em 26/10/2005, aceito para publicação em 14/02/2006. 2 Endereço para correspondência: Universidade São Francisco, Rua Alexandre Rodrigues Barbosa, 45-Centro, Itatiba, SP, CEP: 13251-900 E-mail: anna.villemor@saofrancisco.edu.br
}

uma predição do que uma pessoa fará em uma dada situação (Cattell, citado por Hall, Lindzey \& Campbell, 2000). Para Weiner (2000), a personalidade diz respeito à natureza da pessoa, definida pelas necessidades, atitudes, conflitos e preocupações que influenciam o modo como pensa, sente e age em momentos específicos e em circunstâncias particulares.

No contexto organizacional, a seleção de profissionais com base em avaliações psicológicas é uma prática freqüente e segundo Borman, Hanson e Hedge (1997), as organizações que não utilizam os processos seletivos, incluindo entrevistas e testes, apresentam quedas reais na produtividade, o que su- 


\section{Marcia Eloisa Avona Ferreira}

gere a necessidade dos empregadores demonstrarem maior acuidade na seleção de seus funcionários.

Muitos são os recursos utilizados para avaliação psicológica no contexto de seleção de pessoal, tais como entrevistas, provas de aptidão e testes de personalidade e a escolha dos métodos depende dos objetivos específicos da avaliação. Os dados obtidos podem ser considerados previsores de comportamentos futuros e as decisões tomadas provêm de uma combinação dos vários elementos observados. Cada técnica auxilia as demais fornecendo amplo conjunto de informações sobre o candidato, e a meta é encontrar o melhor preditor para o desempenho futuro (Chiavenato, 2002). Para se avaliar a personalidade, com a finalidade de conhecer mais sobre a pessoa e fazer previsões mais corretas, é freqüente o uso de técnicas projetivas, dentre elas o Zulliger.

Outra prática importante no contexto organizacional é a avaliação de desempenho que compreende um conjunto de normas e procedimentos utilizados pelas organizações para aferir o nível de produtividade dos funcionários. Ela possibilita realizar uma apreciação sistematizada da execução das tarefas, e das responsabilidades do indivíduo, bem como seu potencial futuro. São vários os objetivos da avaliação de desempenho, mas os principais se referem à distinção de programas administrativos, correção das deficiências ou melhoria do desempenho dos funcionários. Também se mostra útil no planejamento e estabelecimento de expectativas, metas e padrões de desempenho acordados entre chefes e subordinados, além de auxiliar na estimativa do potencial, tomada de decisões como promoções, rebaixamentos, demissões, e transferências (Saldanha, 1975; Dewes, 1981; Oliveira-Castro, 1994; Chiavenatto, 1998; Siqueira, 2002).

Avaliação de desempenho pode também oferecer subsídio na seleção de pessoal, representando papel importante no controle de validade do processo seletivo utilizado, uma vez que "na seleção de pessoal está-se fazendo uma inferência ou uma estimativa por antecipação de um determinado desempenho futuro" (Bergamini, 1988). Estando os processos seletivos intimamente relacionados com a avaliação de desempenho, justifica-se o interesse em verificar a validade de um instrumento usado em seleção a partir das avaliações de desempenho realizadas um tempo mais tarde com os candidatos selecionados.
Como mencionado, o Teste de Zulliger é usado com bastante freqüência nessa área, embora não existam pesquisas publicadas que demonstrem especificamente sua validade para o fim de seleção. Tampouco se encontram pesquisas em nosso meio que utilizem o Zulliger sob a perspectiva do Sistema Compreensivo, apesar dessa abordagem ser adotada em outros países ( Mahmood, 1990; Mattlar, Sandhal, Lindberg \& Lethinen ,1990) e da existência de estudos recentes no Brasil que visam normatizar e buscar indicadores de validade nessa perspectiva (Villemor-Amaral, Primi \& Cardoso, 2005).

Apesar de diversas pesquisas envolvendo o teste de Zulliger no Brasil, encontram-se poucos estudos focados no trabalho e na profissão. Dentre eles há os de Van-Kolck (1966) que investigou crises de identidade entre candidatos ao sacerdócio -estudantes de filosofia, e não-candidatos - estudantes de Medicina e Psicologia e de Siminovich e Fachel (2000) que desenvolveram pesquisa para verificar ansiedade e depressão em policiais civis.

Mais especificamente, relacionando personalidade e desempenho, Vaz (1996) enfocou questões relativas à atividade profissional e à cultura. Foram abordados a capacidade de produção, a interação social, e o controle racional em adolescentes e adultos, e os resultados mostram que a média de respostas apareceu mais elevada nos sujeitos de categorias profissionais com instrução superior formal completa ou incompleta. Não houve diferença significativa entre as categorias ocupacionais quanto à capacidade de relacionamento e integração interpessoal, mas quanto à interação social, ela foi melhor entre os das mesmas categorias profissionais o que levou o autor a discutir a questão da influência do "background" cultural mais próximo do sujeito.

Neves (2002) realizou estudo enfocando aspectos da personalidade do motorista de ônibus urbano da Região Metropolitana de Recife, com registros profissionais de conflitos interpessoais, impaciência e transgressões no trânsito ou não. Os resultados confirmaram a hipótese de que os motoristas com antecedentes de transgressão, conflitos e impaciência apresentam sinais indicativos de hostilidade e propensão a reações emocionais descontroladas.

Becker e Vaz (2002) investigaram a criatividade, abstração, raciocínio e ansiedade na personali- 
O Teste de Zuligger e avaliação de desempenho 369

dade de alunos de Arquitetura e Urbanismo, Ciência da Computação e Psicologia. Os resultados mostraram que a ansiedade situacional influencia na mobilização e desencadeamento da criatividade, havendo diferenças significativas entre os três grupos, sendo os estudantes de Psicologia os mais ansiosos e ao mesmo tempo os mais criativos. Não houve diferenças no uso do raciocínio lógico e a inteligência abstrata entre os grupos, mas isto aparece na expressão dos sentimentos, afetos e relacionamento interpessoal, sendo que o curso de Psicologia foi o que mais apresentou este indicador no Zulliger.

Embora não diretamente relacionado ao contexto organizacional, mas com achados significativos para a área, registra-se ainda o trabalho de Primi, Guntert e Alchieri (2002) que desenvolveram um estudo correlacional entre as variáveis do Zulliger e habilidades cognitivas, utilizando as baterias nas quais são encontradas, com maior freqüência, os melhores desempenhos, quais sejam, Provas de Raciocínio (BPR-5), o R1 e o TNRV. Neste estudo foi verificado que: a) os indicadores de inteligência no Zulliger estão um número de respostas acima da média; b) a distribuição de escores de localização G, D, Dd, e S, tem elevação de G; c) uma freqüência acima da média de respostas $\mathrm{M}$ bem vistas; $\mathrm{d}$ ) porcentagem de $\mathrm{F}+$ acima da média e finalmente maior diversidade de conteúdo.

Dada a escassez de estudos específicos de validade do Zulliger, o objetivo deste estudo foi buscar evidências de validade dele por meio da correlação entre os resultados nele e os que o indivíduo obteve na avaliação de desempenho feita posteriormente por seu chefe. Com isso pretendeu-se verificar quais características no Zulliger podem estar associadas aos critérios de bom desempenho de profissionais da área de exatas.

\section{Método}

Participantes: Esta pesquisa foi realizada com base em um banco de dados de uma empresa de telecomunicações. Teve-se acesso aos protocolos dos testes Zulliger de 86 colaboradores que passaram por um processo de seleção, e aos relatórios de avaliação de desempenho desses funcionários, realizada por seus chefes entre seis e doze meses após sua admissão. Todos os participantes tinham graduação completa ou pós-graduação nas seguintes áreas de exatas: Enge- nharia da Computação, Análise de Sistemas, Bacharelado em Ciência da Computação, Engenharia Elétrica, Engenharia Eletrônica e Estatística. Os participantes eram tanto do gênero masculino quanto feminino, sendo superior o número de homens $=54$ (62,7\%), em relação ao de mulheres $=32(37,3 \%)$. As idades variaram de 22 a 43 anos ( $M=30$ anos e 7 meses, $\mathrm{DP}=5$ anos e 3 meses), (moda ficou em 26 anos).

Instrumentos: As análises foram feitas a partir de dados obtidos por meio do Teste de Zulliger, composto de três cartões com manchas de tinta, sendo solicitado ao participante que diga com o que elas se parecem, o que poderiam ser- e de um Questionário de Avaliação de Desempenho desenvolvido pela empresa na qual essa pesquisa foi realizada, que tem por objetivo investigar o desempenho profissional nos itens: Relacionamento Interpessoal; Presença de Atuação Interna e Externa; Tomada de Decisões Oportunas; Competência na Especialização; Busca por Aprimoramento e Inovação; Absorção, Organização, Registro e Divulgação de Conhecimento; Organização; Relacionamento com Pares e Colegas; Solução de Problemas; Orientação para o Cliente.

\section{Procedimentos}

Coleta de dados -Os protocolos dos testes de Zulliger provinham de um banco de dados da empresa e foram obtidos a partir de aplicações individuais $^{3}$. Para essa pesquisa, as respostas foram re-classificadas conforme os critérios do Sistema Compreensivo (Exner, 1999; Villemor-Amaral \& Primi, no prelo) e, para se aferir a precisão na classificação, procedeu-se à avaliação por três juízes independentes, que trabalharam com $30 \%$ dos protocolos. Os juízes participantes eram especialistas no Sistema Compreensivo e procederam à classificação sem acesso aos resultados das avaliações de desempenho.

Análise dos dados - Este estudo buscou correlações entre os indicadores do Z-Test, aplicado no momento de seleção, com os resultados obtidos na avaliação de desempenho realizada pelo chefe imediato no mínimo seis meses após sua admissão.

Hipóteses testadas - Foi verificado: 1) Se a relação $\mathrm{EA}$, determinante $\mathrm{D}$ e o índice $\mathrm{X}+\%$ se

\footnotetext{
${ }^{3}$ Aplicações realizadas pela primeira autora, num período de um a dois anos antes da realização desse estudo. trabalho
} 
correlacionavam positivamente com Solução de Problemas, Organização, Tomada de Decisões Oportunas, e com Absorção, Organização, Registro e Divulgação de Conhecimento. 2) Se a Tomada de decisões oportunas tinha correlação com os Indicadores de Controle e Tolerância ao Estresse (Nota D e Nota D Ajustada). 3) Se havia correlação entre o Relacionamento Interpessoal na Avaliação de desempenho e o índice de Movimentos Cooperativos e Conteúdo Humano no Zulliger. 4) Qual a relação entre a proporção W:M com Busca por Aprimoramento e Inovação. Aplicou-se o teste de correlação de Pearson para verificar características de personalidade do teste Zulliger e questionário de Avaliação de Desempenho, e usou-se o programa estatístico SPSS para análise das pontuações, das médias e desvios padrão.

\section{Resultados e Discussão}

Inicialmente, para verificar a precisão dos resultados, fez-se um estudo de concordância de codificação com $30 \%$ dos protocolos de Zulliger sorteados ao acaso, tendo-se obtido um valor médio total de 85,38\%; as discordâncias distribuiram-se da seguinte forma: Localização $(10,08 \%)$, DQ $(0,87)$, Determinantes (22,69\%), FQ (4,64\%), Par (3,84\%), Conteúdo (17,84\%), Z (21,34\%) e Códigos Especiais (15,5\%).

Com relação à primeira hipótese, a Tabela 1 apresenta os resultados obtidos.

Tabela 1: Correlação entre a relação EA, o código de localização D\%, X+\%, ES_ADJ, COP, H e Avaliação de Desempenho.

\begin{tabular}{|l|c|c|c|c|c|c|}
\hline & TDO & AORDC & ORG & SP & RI & BAI \\
\hline EA & $-0,12$ & $-0,17$ & $-0,15$ & $-0,09$ & & \\
\hline D_\% & 0,10 & $0,26^{*}$ & $0,22^{*}$ & 0,21 & & \\
\hline X+_\% & $-0,04$ & 0,15 & $0,33^{* *}$ & $0,21^{*}$ & & \\
\hline ES_ADJ & $-0,04$ & & & & & \\
\hline COP & & & & & $-0,21$ & \\
\hline H & & & & & $-0,01$ & \\
\hline W_M & & & & & & 0,162 \\
\hline
\end{tabular}

Nota.* $\mathrm{p}<0,05$ ** $\mathrm{p}<0,01$ Legenda - TDO: Tomada de Decisões Oportunas; AORDC: Absorção, Organização, Registro e Divulgação de Conhecimento; ORG: Organização; SP: Solução de Problemas; RI: Relacionamento Interpessoal; BAI: Busca por Aprimoramento e Inovação.

Como se pode observar, o EA não apresentou correlação estatisticamente significativa com ne- nhum dos itens da Avaliação de desempenho, porém o código de localização D mostra correlação significativa com Organização, Absorção, Organização, Registro e Divulgação de Conhecimento (AORCD). As respostas D sugerem a presença de objetividade, interesse por trabalhos práticos, concretos, que gerem resultados em curto espaço de tempo. Valores altos de $\mathrm{D}$ indicam atenção a aspectos mais convencionais, denotando pessoas que tendem a se sair melhor em circunstâncias relativamente limitadas e pouco exigentes, nas quais impera a rotina. Entretanto, situações que exijam flexibilidade, imaginação, criatividade e consciência além do que é imediatamente dado, fazem as pessoas demasiadamente convencionais se sentirem pouco à vontade, e não se saírem bem.

A variável $\mathrm{X}+\%$ se correlacionou positivamente com Solução de Problemas (SP) e com Absorção, Organização, Registro e Divulgação de Conhecimento (AORCD). A porcentagem das respostas $\mathrm{X}+$ no Zulliger indica ajustamento convencional, e quanto maior a proximidade dos perceptos do indivíduo, maior a possibilidade de adaptação e comunicabilidade com o meio. Os resultados sugerem que pessoas bem adaptadas conseguem se comunicar de forma estável, gerar condutas que respondem às exigências reais das situações, se organizar melhor e a identificar problemas reais ou potenciais, tomando providências para evitá-los ou solucioná-los.

Relacionado à Tomada de Decisões oportunas, o que se encontrou é que não houve correlação com a nota $\mathrm{D}$, nem com a D Ajustada. A média de freqüência de Notas D e D Ajustadas apresentou equivalência a zero, ou seja, a diferença entre o EA e o ES não ultrapassou 2,5 (Exner \& Sendin, 1999), sendo que a nota $\mathrm{D}=0$ significa a não existência de sobrecarga de ansiedade, tensão, nervosismo ou irritabilidade manifesta para além da capacidade do indivíduo de suportá-las (Weiner, 2000). Esse resultado aponta que a maioria das pessoas pesquisadas lida satisfatoriamente com as pressões do cotidiano, o que é percebido pelo chefe como uma capacidade de apresentar bom desempenho no desenvolvimento das tarefas.

A hipótese de que Movimentos Cooperativos e Conteúdo Humano pudessem se correlacionar positivamente com o item Relacionamento Interpessoal da Avaliação de desempenho não foi confirmada nesta pesquisa. Movimentos Cooperativos indicam interes- 
se em se envolver em relações de colaboração e de apoio mútuo com os outros, e tendência a obter sucesso na sua vida social. As respostas com conteúdo $\mathrm{H}$ informam como o sujeito percebe a si mesmo e aos demais; possíveis distorções nesta percepção estarão presentes na forma de apreender e entender os outros.
A relação W:M indica a proporção de respostas W (gerais) para as M(Movimento Humano), e aponta para o interesse em alcançar metas e realizar ambições; nesta pesquisa os dados não mostram correlação com Busca por Aprimoramento e Inovação. Entretanto, numa análise exploratória dos resultados, foram encontrados outros itens que apresentaram correlações significativas conforme demonstrado na Tabela 2.

Tabela 2: Correlação encontrada entre Z-Test e Avaliação de Desempenho

\begin{tabular}{|c|c|c|c|c|c|c|c|c|c|}
\hline & RI & PAIE & TDO & CE & AORDC & ORG & RPC & SP & OC \\
\hline Lambda & & & & & $0,228 *$ & & $0,239 *$ & & \\
\hline X+_PC & & & & & & $0,331^{* *}$ & & $0,218^{*}$ & \\
\hline D_PC & & & & & $0,265^{*}$ & $0,222^{*}$ & & & \\
\hline Dd_PC & & & & & & & & $0,220^{*}$ & \\
\hline DQV_PC & & & & & & & & $-0,219 *$ & \\
\hline XU_PC & & & & & & & & & $0,346 * *$ \\
\hline$S^{-}$ & & & & & & & & $0,212 *$ & \\
\hline Egocent & & & $0,29 * *$ & & $-0,257^{*}$ & & & & \\
\hline An_Xy & $0,224 *$ & & & & & & & & \\
\hline Sum_FM & & & & $-0,236^{*}$ & & & & & \\
\hline Sum_T & & & & & & & $0,275^{*}$ & & \\
\hline Ma_Mp & & & & & & & & $0,214^{*}$ & \\
\hline Sum_Y & & & & & & & & $0,221 *$ & \\
\hline Sum $\mathrm{MH}$ & & & & $0,216^{*}$ & & & & & \\
\hline EB & & & & $0,28 * *$ & & & & & \\
\hline A & & & & & $0,227 *$ & & & & \\
\hline Art & & $0,320 * *$ & & & & & & & \\
\hline $\mathrm{Fi}$ & & & & & & $0,279 * *$ & & & \\
\hline
\end{tabular}

Nota.* $\mathrm{p}<0,05 * * \mathrm{p}<0,01$

Legenda - RI: Relacionamento Interpessoal; PAIE: Presença de Atuação Interna e Externa; TDO: Tomada de Decisões Oportunas; CE: Competência na. Especialização; AORDC: Absorção, Organização, Registro e Divulgação de Conhecimento; ORG: Organização; RPC: Relacionamento com Pares e Colegas; SP: Solução de Problemas; OC: Orientação ao Cliente.

No teste Zulliger, o Lambda indica de que forma a pessoa foca sua atenção. Se for adequada e adaptada, esta consegue se manter mais consciente dos eventos internos e externos, podendo tolerar ambigüidades e incertezas. Valores baixos sugerem abertura excessiva à experiência, o que implica grande sensibilidade quanto a suas vivências. Pessoas com essa característica tendem a apreciar e até procurar situações ambíguas e complexas, em geral sentindose melhor em ambientes relativamente pouco estruturados e abertos. Já, os valores altos, apontam para uma falta de abertura à experiência, com tendência a perceber o mundo de modo muito restrito, baixa tolerância à ambigüidade e incertezas, e sentindo-se melhor em situações definidas e bem estruturadas, com tendência a optar pelas simples mesmo frente a problemas complexos (Weiner, 2000).

O Lambda se correlacionou positivamente com a Absorção, Organização, Registro e Divulgação de Conhecimento (AORDC) e Relacionamento com Pares e Colegas (RPC) na avaliação de desempenho, e indicou que, à medida que os participantes apresentaram Lambda mais elevado, foram melhor avali- 


\section{Marcia Eloisa Avona Ferreira}

ados nesse ítem. Foi ressaltada de maneira positiva a qualidade em desenvolver trabalhos em equipe de forma cooperativa e participativa para profissionais com essas características. Esse dado contraria em parte as expectativas, mas sugere que entre os profissionais da área de exatas certo distanciamento de envolvimentos mais pessoais ou maior formalismo é apreciado e facilita bons contatos.

As respostas de localização D no Zulliger se correlacionaram positivamente com Absorção, Organização, Registro e Divulgação de Conhecimento (AORDC), além da Organização (ORG) já citada, o que significa que o aumento da quantidade de respostas desse tipo leva a melhores avaliações dos superiores a respeito de seus subordinados.

As respostas do tipo Dd obtiveram uma correlação negativa com o item Solução de Problemas (SP); isto demonstra que o foco excessivo sobre o que é incomum, pouco usual, faz com que a pessoa desconsidere o que é obvio, posicionando-se de maneira pouco convencional e deixando-se seguir por suas idiossincrasias, ou seja, por aquilo que lhe é mais pessoal. Esse tipo de correlação é compreensível, pois pessoas extremamente detalhistas, voltadas para a própria maneira de perceber a realidade, tendem a ser cuidadosas, perfeccionistas e conseqüentemente mais lentas no exercício de suas tarefas, levando mais tempo para concluir um trabalho ou encontrar soluções para problemas.

Também houve correlação negativa entre respostas DQV e solução de problemas na avaliação de desempenho. Embora tenham ocorrido poucas respostas DQV entre os participantes, por serem profissionais mais lógicos, esse fato se mostra coerente, pois a freqüência de respostas vagas no Zulliger denota forma de pensar mais primitiva e imatura, ou seja, pouco elaborada, com tendência a evitar o compromisso com o que é percebido, e menor eficiência na proposta de solução de problemas.

Pessoas com Xu\% elevado tendem a maior individualismo na maneira de ver o mundo e a serem mais ousadas nos caminhos que escolhem; isto indica, de modo geral, maior desadaptação devido a falta de convencionalismo. Os dados apontam que os níveis mais altos de $\mathrm{Xu} \%$ não correspondem a uma boa avaliação de desempenho no que se refere a atender às expectativas dos clientes internos e externos.
Nesta pesquisa, o traço XU\% do teste Zulliger se correlacionou negativamente com Orientação para o Cliente (OC).

Um outro traço de personalidade que se correlacionou negativamente com o desempenho em Solução de Problemas (SP) foi um oposicionismo elevado (S). As respostas do tipo $S$, quando em excesso, indicam tendências de oposição generalizada, que extrapolam a autonomia adaptativa, e estão associadas a sentimentos subjacentes de raiva e ressentimento. Os resultados indicam que à medida que aumentam as S, pior é a avaliação dos supervisores quanto a identificar problemas e buscar soluções eficazes para eles.

Outra correlação negativa encontrada diz respeito ao índice de egocentrismo e a Tomada de Decisões Oportunas (TDO) e Absorção, Organização, Registro e Divulgação de Conhecimento (AORDC). Os dados mostram que se esse índice aumenta no Zulliger, piores são os resultados das avaliações realizadas pelos supervisores nos itens acima. O índice de egocentrismo é uma medida da proporção em que o indivíduo volta sua atenção para si, preocupando-se mais consigo mesmo do que com os demais.

Encontrou-se ainda uma correlação positiva entre Relacionamento Interpessoal (RI), e presença de respostas anatômicas e radiografia. Curiosamente, o aumento de respostas com estes conteúdos indica que há excessiva preocupação com o corpo, podendo trazer informação sobre alterações da auto-imagem. Em princípio parece difícil compreender o significado da correlação desses indicadores no Zulliger com melhores avaliações no item Relacionamento interpessoal, mas supõe-se que nem sempre dificuldades no contato com os outros, num sentido mais profundo, sejam percebidas no trabalho como negativas.

As respostas FM (movimento de animais) têm uma correlação negativa com Competência na Especialização (CE), da qual se espera habilidade no exercício das atribuições. FM associa-se a uma consciência perturbadora de necessidades que não estão sendo satisfeitas. É o tipo de ideação que aparece quando não se pensa deliberadamente em algo concreto (o pensamento voa, Exner, 1999) sugerindo dificuldade de concentração.

Nesta pesquisa encontrou-se correlação negativa entre a somatória de respostas $\mathrm{T}$ (que significam 
interesse literal em tocar ou se aproximar de alguém, física ou psicologicamente) com o Relacionamento com Pares e Colegas (RPC). A não presença desta característica em um protocolo não indica que a pessoa evite o relacionamento interpessoal, mas tende a ser mais distante e desvinculada do que próxima e íntima. O valor esperado é de uma resposta por protocolo; mais do que uma sugere pessoas com o risco de empreender busca desesperada e indiscriminada de relacionamento íntimo, fazendo com que suas necessidades de contato interpessoal transcendam seu melhor julgamento.

O movimento ativo sugere capacidade de tomar iniciativa e solucionar problemas, além de manter a de pensar de modo flexível, podendo contemplar perspectivas alternativas de suas experiências, e mudar os pontos de vista, sendo que a superação de movimentos ativos sobre os passivos levaria a pensar de forma construtiva. Já o contrário indica forte presença de fantasias no pensamento, o que compromete o ajustamento. Houve correlação positiva com Absorção, Organização, Registro e Divulgação de Conhecimento (AORDC).

A presença de respostas $Y$ indica dificuldade de condutas mais ajustadas na solução de problemas, uma vez que esta característica se correlacionou negativamente com este item na avaliação de desempenho. De todos os participantes, $48,8 \%$, portanto um número significativo forneceu ao menos uma resposta do tipo Y, que indica a presença de estresse situacional, com sentimentos de desproteção, desamparo e impotência que podem chegar a provocar um efeito paralisador sobre as condutas.

A somatória de respostas de movimento humano (M) se correlacionou negativamente com Competência na Especialização (CE). A presença de movimento humano implica algum tipo de projeção, e pressupõe uma criação, uma vez que é o único determinante que não existe nas pranchas, ou seja, há uma criação mental de características que são atribuídas ao campo de estímulo. As respostas de movimento humano estão relacionadas, entre outras, à imaginação, campo de relacionamentos interpessoais, autoconceito e atividade ideacional deliberada. O item avaliado no desempenho pode estar considerando que se está valorizando os pensamentos concretos e práticos em detrimento daqueles mais fantasiosos e cri- ativos ao demonstrar competência no exercício das atribuições que são conferidas ao funcionário, nessa área de especialização, onde talvez a competência prática e técnica contem mais que a imaginação e criatividade.

Os resultados indicam que os extratensivos tenderam a apresentar um melhor resultado quando avaliados em Competência na Especialização. Isso pode ocorrer em função da tendência da pessoa extratensiva ser mais comunicativa e espontânea, o que facilita demonstrar aos demais o que pensa ou sente.

As respostas de conteúdo animal se correlacionaram positivamente com Absorção, Organização, Registro e Divulgação de Conhecimento (AORDC), indicando que pessoas que tenham apresentado mais respostas com conteúdo A em seus protocolos foram melhor avaliadas por seus gerentes neste quesito.

O conteúdo Arte (Art) apresentou correlação positiva com Presença de Atuação Interna e Externa (PAIE). A presença de Arte nas respostas está relacionada ao nível de intelectualização da pessoa, ou seja, da tendência de tentar neutralizar as emoções por meio do raciocínio, buscando a lógica para explicar no lugar de vivenciar os sentimentos. Esse resultado sugere que foram avaliados positivamente os profissionais que são mais racionais, e que buscam explicação para os fatos, não vivenciando-os emocionalmente. Considerando-se que esse item da avaliação de desempenho se refere à realização de apresentações formais em nome da empresa para tratar de assuntos relacionados a atividade desses profissionais, tais resultados não surpreendem já que a amostra é composta de profissionais técnicos, altamente especializada em sua área de atuação.

A presença do conteúdo fogo correlacionouse negativamente com Organização (ORG) na avaliação de desempenho, indicando que pessoas impulsivas podem ser menos organizadas em suas tarefas de planejar, distribuir adequadamente o tempo e os recursos em relação às responsabilidades assumidas.

Resumindo, os dados levantados indicaram que o item Organização na Avaliação de Desempenho se correlacionou positivamente com o determinante $\mathrm{D}$, e $\mathrm{X}+\%$, e negativamente com o conteúdo Fogo. O que indica que para o avaliador a pessoa organizada é aquela que é mais prática, objetiva e bem ajustadas 


\section{Marcia Eloisa Avona Ferreira}

às necessidades do ambiente, além de moderada, sem demonstrações de impulsividade nas atitudes.

O item Absorção, Organização, Registro e Divulgação de Conhecimento apresentou correlação positiva com Lambda, com determinante D e com conteúdo Animal, porém foi negativa a correlação com egocentrismo. Este item contempla a expectativa de que o avaliado possa assimilar novos conhecimentos, e os organize de forma prática, registrando-os posteriormente na forma de relatórios técnicos que permitam futuras consultas por parte de qualquer integrante do grupo, o que justifica a baixa correlação com egocentrismo.

O item Relacionamento com Pares e Colegas também apresentou correlação positiva com o Lambda, já discutido anteriormente, e negativa com Sum T. O excesso de $\mathrm{T}$ indica uma busca desesperada e indiscriminada de relacionamento íntimo, nas quais as necessidades de contato interpessoal podem transcender o melhor julgamento. A correlação negativa encontrada é coerente com o fato de que se espera que a pessoa possa desenvolver trabalhos em equipe mantendo uma postura profissional participativa e colaboradora, menos carregada de carências que venham a interferir nos relacionamentos.

Esses dados sugerem que os avaliadores tendem a pontuar melhor neste item as pessoas que são menos rígidas ou até mesmo as mais inflexíveis e pouco emocionais, em detrimento daquelas que são claramente imaturas ao estabelecer vínculos em seus relacionamentos com pares e colegas.

O item Orientação para o Cliente avalia a capacidade de adquirir novos conhecimentos no campo de atuação, de modo a usá-los para melhorar produtos, serviços e processos, atendendo às necessidades e expectativas dos clientes internos e externos. Este se correlacionou negativamente com Xu\%, o que é coerente com o fato de que pessoas com foco no cliente devem estar mais atentas às necessidades e expectativas destes.

No item Relacionamento Interpessoal houve correlação com respostas $\mathrm{F}$, além de respostas de conteúdos anatômicos e radiografia. As respostas $\mathrm{F}$ indicam ajustamento perceptivo ou controle intelectual, de forma a focalizar a atenção, os sistemas de controle e juízos discriminantes na relação com os padrões do mundo real. Os resultados então sugerem que as melhores avaliações em Relacionamento Interpessoal nessa amostra correspondem a pessoas centradas, mas não necessariamente mais maduras quanto a auto-imagem num sentido mais profundo.

No item Competência na Especialização, as correlações com FM e M foram negativas; embora haja maior freqüência de sujeitos introversivos, os extratensivos foram melhor avaliados nesse quesito, o que pode ter ocorrido pelo fato de as pessoas extratensivas serem mais alegres, comunicativas e espontâneas, o que facilita demonstrar aos demais o que pensam ou sentem.

O item Solução de Problemas denota iniciativa para prever ou identificar problemas reais ou potenciais, analisando causas e conseqüências, buscando todas as formas de respostas, tomando providencias para evitá-los e/ou solucioná-los. Esse item apresentou correlações positivas com $\mathrm{X}^{+}$, com movimento ativo e correlações negativas com Dd\%, DQV\%, S e Sum_Y. Compreende-se tal resultado pela justificável valorização da capacidade de tomar iniciativa e solucionar problemas de modo condizente com o dado de realidade, bem como pela apreciação negativa de comportamentos idiossincráticos detalhistas, lentidão, descompromisso com a objetividade e excesso de ansiedade.

O item Presença de Atuação Interna e Externa avalia a habilidade para realização de apresentações formais, participação em discussões, dentro ou fora da organização, em assuntos relativos à atividade. Houve correlação positiva deste item só com conteúdo Arte; isto é coerente tendo em vista que a atividade dos profissionais da amostra é essencialmente técnica, muito uso do intelecto e menores chances para vivenciar os sentimentos.

\section{Considerações Finais}

Esse estudo trouxe indicadores de validade para o uso do Zulliger, Sistema Compreensivo no processo de seleção de profissionais da área de exatas uma vez que se constatou a presença de indicadores com correlações significativas com as avaliações de desempenho e permitiu verificar que os profissionais de exatas bem avaliados são aqueles que apresentaram tendência a maior formalidade nos relacionamentos, reserva social, preferência por atividades de execução solitária, de cunho intelectual, mecânico ou ar- 
tístico. São também valorizados os que se mostraram mais voltados ao sentido prático das coisas, a sua funcionalidade e utilitarismo, além de se comportarem de maneira realista e independente. Por outro lado, como as correlações significativas encontradas são baixas, é importante ressaltar que são muitos os fatores envolvidos no processo de avaliação de desempenho, o que estimula a continuidade de estudos nessa área.

\section{Referências Bibliográficas}

Becker, M.A. \& Vaz, C. E. (2002). Criatividade, abstração, raciocínio lógico, ansiedade e sua interação na personalidade de estudantes de Arquitetura, Computação e Psicologia. Psicodiagnosticar, 12(1), 31-40.

Bergamini, C. W. (1988). Avaliação de desempenho humano na empresa. São Paulo: Atlas.

Borman, W. C.; Hanson, M. A. \& Hedge, J.W. (1997). Personnel Selection. Annual Reviews of Psychology. Califórnia. 48, 299-337.

Chiavenato, I. (1998). Desempenho Humano nas Empresas. Como desenhar cargos e avaliar o desempenho. São Paulo: Atlas.

Chiavenato, I. (2002). Recursos Humanos. Edição Compacta. São Paulo: Atlas.

Dewes, F. (1981). Fatores de avaliação do desempenho no trabalho. Psico 2(1), 56-60.

Exner, J. E. Jr. (1999). Manual de Classificação do Rorschach - Sistema compreensivo.São Paulo: Casa do Psicólogo.

Exner, J. E. Jr. \& Sendín, C. (1999). Manual de Interpretação do Rorschach para o sistema compreensivo. São Paulo: Casa do Psicólogo.

Hall, C. S.; Lindzey G. \& Campbell J. B. (2000). Teorias da Personalidade. Porto Alegre: Artes Médicas.

Mahmood, Z. (1990). The Zulliger Test: Its past and Future. British Journal of Projective Psychology, 35(2), 2-16.

Mattlar, C. E.; Sandahl, C.; Lindberg, S. \& Lehtinen, V. (1990). Methodological issues associated with the application of the comprehensive system when analysing the Zulliger, and the structural resemblance between the Zulliger and the Rorschach. British Journal of Projective Psychology, 35(2), 17-27.

Neves, M.F.S. (2002). Reações emocionais, contexto de trabalho e saúde mental de motoristas de ônibus. Dissertação de Mestrado em Psicologia. Pontifícia Universidade Católica do Rio Grande do Sul. Porto Alegre.

Oliveira-Castro, G. A. (1994). Avaliação de desempenho em psicologia: questões conceituais e metodológicas. Psicologia:Teoria e Pesquisa,3(10), 355-374.

Pasquali, L. (2001). Técnicas de Exame Psicológico - TEP Vol. I: Fundamentos das Técnicas Psicológicas. São Paulo: Casa do Psicólogo / Conselho Federal de Psicologia.

Primi, R, Guntert, A. E. V. \& Alchieri, J. C. (2002). Um estudo correlacional entre a bateria de provas de raciocínio com o teste de Zulliger. Psico, 33(1), 53-76.

Saldanha, G. S. (1975). Manual de Seleção de Pessoal. Rio de Janeiro: Livros Técnicos e Científicos Editora S.A.

Siminovich, M. \& Fachel, J. M. G. (2000). Técnica de Zulliger - teste-reteste, ansiedade e depressão em policiais do interior e capital do Rio Grande do Sul. Em C. E. Vaz. II Congresso Nacional da Sociedade Brasileira de Rorschach e outros métodos projetivos. (p. 284-288). Porto Alegre: AGE Editora.

Siqueira, W. (2002). Avaliação de desempenho: como romper amarras e superar modelos ultrapassados. Rio de Janeiro: Reichmann \& Affonso.

Van Kolck, T. (1966). Um estudo com estudantes ao sacerdócio com o Z-Test. Revista de Psicologia Normal e Patológica,12(1-2), 61-81.

Vaz, C. E. (1996). Multiethnic culture, Professional success, performance and inferiority, through Rorschach and Z-Test. XV International Congress of Rorschach \& Projective Techniques. Boston. 8-12. 
376 Marcia Eloisa Avona Ferreira

Villemor-Amaral A. E; Primi, R. \& Cardoso, L.M. (2005). The Zulliger Test and the Comprehensive System in a Brazilian Sample. Anais do XVIII Interantional Rorschach Congress, Barcelona.

Villemor-Amaral, A. E. \& Primi, R. (no prelo). Manual de codificação e interpretação do Zulliger no Sistema Compreensivo.

Weiner, I.B. (2000). Princípios da Interpretação do Rorschach. São Paulo: Casa do Psicólogo. 